\section{Usos teórico-metodológicos das pesquisas na área de Ciências Sociais e Humanas em Saúde}

\author{
Theoretical and methodological uses of research in \\ Social and Human Sciences in Health
}

\begin{abstract}
The current article aims to map and critically reflect on the current theoretical and methodological uses of research in the subfield of Social and Human Sciences in Health. A convenience sample was used to select three Brazilian public health journals. Based on a reading of 1,128 abstracts published from 2009 to 2010, 266 articles were selected that presented the empirical base of research stemming from Social and Human Sciences in Health. The sample was classified thematically as "theoretical/ methodological reference", "study type/ methodological design", "analytical categories", "data production techniques", and "analytical procedures". We analyze the sample's emic categories, drawing on the authors' literal statements. All the classifications and respective variables were tabulated in Excel. Most of the articles were self-described as qualitative and used more than one data production technique. There was a wide variety of theoretical references, in contrast with the almost total predominance of a single type of data analysis (content analysis). In several cases, important gaps were identified in expounding the study methodology and instrumental use of the qualitative research techniques and methods. However, the review did highlight some new objects of study and innovations in theoretical and methodological approaches.
\end{abstract}

Social Sciences; Humanities; Research
Suely Ferreira Deslandes 1

Jorge Alberto Bernstein Iriart ${ }^{2}$

\section{Introdução}

O presente artigo visa a mapear e refletir criticamente sobre o estado de arte dos usos teórico-metodológicos correntes em pesquisas do subcampo das Ciências Sociais e Humanas em Saúde.

Entendemos que a forma como as pesquisas são metodologicamente construídas reflete a trajetória de constituição das Ciências Sociais no campo da Saúde Coletiva no país e guarda as influências de diversos atores, lideranças acadêmicas históricas ou emergentes, e além das influências institucionais e de suas respectivas culturas científicas. Mais do que uma escolha individual, de expertise douta, o exercício me todológico se dá num campo científico, como afirma Bourdieu 1, com suas intrínsecas relações de força, de monopólio e de lutas. O que sempre estará em jogo, por fim, de maneira inseparável, é a capacidade técnica e o poder social de falar e agir de maneira autorizada e com autoridade sobre nossos objetos e temas, como um agente legitimado pela ciência.

Não nos prestamos a uma análise de mérito das pesquisas analisadas, o que seria pretensioso e descabido em face do nível de consolidação deste subcampo. As Ciências Sociais e Humanas em Saúde integram o campo da Saúde Coletiva há pelo menos 50 anos. São responsáveis pela inclusão inovadora de inúmeros temas oriundos das agendas investigativas das disciplinas que as 
compõem, assim como propiciaram uma análise, interna ao campo, de seus modelos de produção de conhecimentos e de cuidados. Têm, ainda, produzido reflexões ajustadas aos desafios impostos pelas transformações sociais contemporâneas, e sua incursão também colabora diretamente para ampliação do escopo de análise política do campo e pela formação de pesquisadores, seja na graduação, seja na pós. Trata-se de uma "área" que, a julgar pelos últimos congressos específicos ao subcampo, agrega milhares de praticantes ou simpatizantes $2,3,4$.

Buscamos identificar as tendências metodológicas correntes e apontar as limitações impostas pela hegemonia de certos modos de interrogar a realidade, de produzir os dados e de interpretá-los. Ao fazê-lo, evitamos dissociar os instrumentos intelectuais da sua utilização nas pesquisas reais, que, por sua vez, refletem a historicidade deste campo 5 .

Trabalhamos com o que pesquisadores consideraram importante informar num artigo científico, isto é, num espaço estruturado de comunicação cuja elasticidade de regras poderá depender dos agentes envolvidos e de seu capital acadêmico (p.ex., o perfil que os editores desejam imprimir à revista num mercado científico, se o autor é mais ou menos renomado ou experiente).

A reflexão pretende contribuir para a problematização do fazer pesquisa, de seus domínios de competência técnica e também dos modos de reconstruir a realidade social por meio de um conjunto de artefatos teóricos e conceituais. Em última instância, buscamos contribuir para o necessário exercício de vigilância epistemológica de cada pesquisador, voltados à explicitação e ao confronto contínuo das suas operações científicas e dos pressupostos implicados; comprometidos com reflexão autocrítica acerca das condições e limites de validade de técnicas, conceitos e teorias empregadas e, consequentemente, do conhecimento que se constrói 5.

\section{Métodos}

Trabalhamos com uma amostra de conveniência dos periódicos brasileiros que têm como missão a publicação de artigos da área de Saúde Coletiva. Todos os periódicos eleitos contam mais de dez anos de existência, estão vinculados a importantes bases de indexação nacionais e internacionais, são classificados nos estratos mais altos do Qualis da Coordenação de Aperfeiçoamento de Pessoal de Nível Superior (Capes), selecionam artigos valendo-se da revisão por pares, apresentando elevado nível de critérios para publicação.
Dois periódicos publicam material de escopo diversificado, acolhendo os três principais campos disciplinares da Saúde Coletiva (Cadernos de Saúde Pública - CSP e Ciência e Saúde Coletiva - CSC); o terceiro volta-se para a divulgação da produção das Ciências Sociais, Humanas e Filosofia em Saúde (Interface: Comunicação, Saúde, Educação - INTERF).

Com o objetivo de abarcar a produção recente do campo, selecionamos o período de 20092010. Mesmo sem garantir a representatividade estatística do acervo de revistas científicas que publicam artigos das Ciências Sociais e Humanas em Saúde, tal escolha permitiu-nos uma robusta base empírica de apoio à reflexão: 46 números regulares e oito suplementos.

Assim como em outros estudos que buscavam delimitar o escopo de abrangência das Ciências Sociais e Humanas em Saúde numa dada produção bibliográfica 6 , neste trabalho não foram incluídos os artigos das áreas de políticas, planejamento e gestão e de epidemiologia. Os artigos das seções de "debates", de "opinião” e de "fórum" não foram, igualmente, integrados ao corpo do estudo, por não constituírem divulgação de resultados de pesquisa e por serem publicados a convite do editor, não com base em seleção de peer review. Todos os trabalhos publicados nas seções voltadas aos "artigos originais" foram contabilizados. Os artigos de ensaio teórico e os de surveys, embora contabilizados, não participaram da análise. Os artigos aqui analisados foram exclusivamente aqueles que possuíam uma base empírica de investigação com aporte nos métodos oriundos das Ciências Sociais e Humanas em Saúde.

O processo de seleção dos artigos deu-se a partir da leitura dos 1.128 resumos dos trabalhos publicados pelos referidos periódicos, incluindo todos os números regulares e suplementos. A leitura dos títulos e das palavras-chave serviu de forma coadjuvante ao processo classificatório.

A classificação de um texto como pertencente ou não ao campo das Ciências Sociais e Humanas em Saúde foi realizada independentemente por dois pesquisadores; quando havia discordância, um terceiro investigador era solicitado a classificar o artigo. Todos os pesquisadores envolvidos nesta codificação possuem formação de graduação e/ou pós-graduação na área de Ciências Sociais. Uma vez selecionado, cada artigo foi analisado considerando-se as seções de "introdução" e "metodologia”. O acervo, composto de 266 artigos, foi classificado a partir das temáticas "referencial teórico-metodológico", "tipo de estudo-desenho metodológico”, “categorias analíticas utilizadas", "técnicas para a produção de dados" e "procedimentos de análise”. As categorias êmicas 
do acervo foram analisadas com base nas declarações literais dos autores. Todas as classificações e respectivas variáveis foram tabuladas em Excel (Microsoft Corp., Estados Unidos).

A base descritiva da análise não pode ser vista como evidência positivista, mas como suporte interpretativo ao debate traçado, constituindo provocações reflexivas, naturalmente sujeitas a outras leituras.

\section{Resultados e discussão}

No acervo analisado, 36\% (407) dos artigos publicados nas seções de divulgação de pesquisas pertencem ao campo disciplinar das Ciências Sociais e Humanas em Saúde (Tabela 1). Como se verificou, dois dos três periódicos apresentam destacada publicação na área; por sua vez, a revista INTERF possui missão editorial voltada também para a área em questão. Estudo com acervo representativo dos periódicos de Saúde Coletiva no triênio (2004-2006) demonstra uma participação bem menor dos artigos de Ciências Sociais e Humanas em Saúde na totalidade de publicações (21\%) 6 .

Chama a atenção a boa receptividade das revistas aos artigos que constituem ensaios teóricos, pois, além daqueles incluídos a convite dos editores, 7,5\% dos trabalhos publicados após o processo seletivo pertencem a esta categoria. Os surveys em Ciências Sociais e Humanas em Saúde, ainda que em menor número, apontam as potenciais contribuições das análises quantitativas de base sociológica.

Considerando-se o tipo de estudo declarado pelos autores, a maioria se autodenominou como "qualitativo" (44,6\%) e "triangulação de métodos" ou "estudo quantiqualitativo" foi a designação de 7,8\%. Essa definição geral poderia constituir a única classificação do estudo ou vir agregada a outra, de cunho mais específico. Do acervo analisado, artigos sem quaisquer definições de tipo de estudo (17,3\%) se destacam, revelando fragilidade na exposição da metodologia da pesquisa.

Mais específicos em suas definições, encontramos o predomínio de estudos que se apresentam como "exploratórios"/ "descritivosexploratórios”/“descritivos” (16,5\%). Vale problematizar tal achado, pois geralmente a classificação de "exploratório" é dada à primeira fase do ciclo da pesquisa, destinada à construção do objeto. Portanto, uma pesquisa exploratória seria totalmente dedicada à construção de conhecimentos sobre tema ainda desconhecido, permitindo a produção de hipóteses de leitura daquela realidade 7,8 . Por outro lado, cabe o estranhamento a respeito de um artigo de Ciências Sociais que se designe apenas como "descritivo", sem se comprometer com a produção de inferências interpretativas.

Os artigos definidos pelos autores como "etnografias" ou "de base ou cunho etnográfico" também se destacaram no acervo, somando 34 trabalhos (12,7\%). Observou-se grande diversidade de campos etnográficos, com destaque para a etnografia realizada em diferentes serviços de saúde (11 artigos). Alguns estudos foram realizados em comunidades ou bairros (7 artigos), por vezes enfocando espaços ou grupos específicos, como uma feira livre ou pessoas em situação de rua; duas etnografias foram realizadas em aldeias indígenas. Determinadas instituições

Tabela 1

Distribuição de artigos de Ciências Sociais e Humanas em Saúde segundo periódico, 2009-2010.

\begin{tabular}{|c|c|c|c|c|c|c|c|}
\hline \multirow[t]{2}{*}{ Periódico/Ano } & \multirow{2}{*}{$\begin{array}{l}\text { Total de } \\
\text { artigos }\end{array}$} & \multicolumn{2}{|c|}{$\begin{array}{c}\text { Artigos em Ciências } \\
\text { Sociais e Humanas } \\
\text { em Saúde }\end{array}$} & \multicolumn{2}{|c|}{ Ensaios } & \multicolumn{2}{|c|}{ Surveys } \\
\hline & & $\mathrm{n}$ & $\%$ & $n$ & $\%$ & $\mathrm{n}$ & $\%$ \\
\hline CSC/2009 & 192 & 60 & 31,3 & 25 & 13,0 & 5 & 2,6 \\
\hline CSC/2010 & 331 & 101 & 30,5 & 14 & 4,2 & 34 & 10,3 \\
\hline CSP/2009 & 251 & 25 & 10,0 & 2 & 0,8 & 10 & 4,0 \\
\hline CSP/2010 & 188 & 10 & 5,3 & 1 & 0,5 & 5 & 2,7 \\
\hline INTERF/2009 & 101 & 36 & 35,6 & 31 & 30,7 & 2 & 2,0 \\
\hline INTERF/2010 & 65 & 34 & 52,3 & 12 & 18,5 & 0 & 0,0 \\
\hline Total & 1.128 & 266 & 23,6 & 85 & 7,5 & 56 & 5,0 \\
\hline
\end{tabular}

CSC: Ciência e Saúde Coletiva; CSP: Cadernos de Saúde Pública; INTERF: Interface: Comunicação, Saúde, Educação. 
também foram objeto de observação etnográfica, como academias de musculação, clubes sociais, instituições de longa permanência para idosos ou associações de pessoas vivendo com HIV. Três estudos trazem inovação metodológica ao abordarem o ciberespaço, com observação participante on line em comunidades e fóruns virtuais. A observação participante aparece como técnica de produção de dados na grande maioria dos estudos associada a teorias de origem antropológica, sendo citados autores estrangeiros, como, por exemplo, Geertz C, Jackson M, Pina-Cabral J, Becker H, Kleinman A, Good B, Corin E \& Bibeau G, Douglas M, Clifford J, Atkinson P, além de brasileiros e latino-americanos, como Peirano M, Menendez E, Víctora C, Knauth D, Hassen M, Magnani JG, Oliven GR e Oliveira RC.

No entanto, certos estudos, que se denominaram etnográficos, foram baseados unicamente em entrevistas semiestruturadas, sem nenhuma menção à observação participante ou embasamento teórico-epistemológico que fundamentassem uma abordagem etnográfica.

Chama a atenção, na maioria dos estudos etnográficos, a ausência de reflexividade sobre a relação entre o pesquisador e os sujeitos da pesquisa. As questões colocadas pela antropologia pós-moderna 9 , como o questionamento da autoria do texto etnográfico, a multivocalidade e a incorporação da biografia do pesquisador como parte legítima do estudo, não aparecem como preocupação dos autores.

Os 31 artigos $(11,6 \%)$ de estudos de caso representam este consagrado modelo de pesquisa. As demais definições, agrupadas na categoria "outras", reuniram 43 artigos, expressando a bem-vinda diversidade disponível nas Ciências Sociais e Humanas em Saúde. Neste grupo, vale destacar a pouca participação da pesquisa-ação (3 artigos), das de cunho biográfico (biografias e histórias de vida - 3 artigos) e as de groundedtheory (pesquisa fundamentada -2 artigos).

Um total de 124 artigos (46,6\%) define claramente sua filiação a um referencial teórico-metodológico. Observa-se uma grande variedade de perspectivas teórico-metodológicas oriundas de diferentes disciplinas no campo das Ciências Sociais e Humanas. Os demais artigos utilizam teorias de médio alcance focadas em objetos específicos, sem referenciar as incursões analíticas nos universos simbólicos que se propõem analisar a teorias de suporte.

As representações sociais continuam sendo utilizadas pelos pesquisadores da área, valendose de diferentes definições e associadas a perspectivas epistemológicas e metodológicas distintas. Alguns trabalhos utilizam o conceito com base em seu desenvolvimento na antropologia/ sociologia (Magnani JC, Queiroz M, Minayo MC), e outros na perspectiva da psicologia social (Jodelet D, Moscovici S, Guareschi PA, Spink MJ).

A abordagem hermenêutica é citada em diversos artigos com diferentes enfoques (hermenêutica crítica, hermenêutica dialética, ou, simplesmente, hermenêutica), fazendo referências a filósofos como Gadamer HG e Ricoeur P. Outros estudos referem a antropologia interpretativa crítica, alguns associados ao sistema de signos, significados e práticas. Na hermenêutica crítica, são citados antropólogos como Geertz C, Kleinman A, Corin E \& Bibeau G; na hermenêutica dialética, a principal referência é Minayo MC. Alguns estudos se vinculam a diversas correntes da fenomenologia: abordagem fenomenológica heideggeriana, fenomenologia de Merleau Ponty, fenomenologia-pensamento rogeriano humanista. Este é o caso, também, de estudos de narrativas e experiência da enfermidade, os quais se baseiam em autores como Bury M, Hyden LC, Kleinman A, Csordas T, Thompson JB, Good B e Alves P \& Rabelo M e utilizam conceitos como experiência da enfermidade, narrativas e corporeidade (embodiment). Percebe-se, nos estudos hermenêuticos e fenomenológicos, a crescente influência da produção da antropologia e sociologia médicas anglo-saxônicas.

Outro grupo de estudos reúne aqueles que se definem como abordagem construtivista/construcionismo social, ou que trabalham com diferentes formas de análise do discurso (Análise do Discurso, Análise Crítica de Discurso; Semiologia dos Discursos Sociais, Abordagem Discursiva Sistêmica), tendo como referências autores como Foucault M, Denzin N \& Fairclough N, Bahktin M, Gergen K, Spink MJ e Medrado B.

Por fim, identificamos uma longa lista de perspectivas teóricas de diferentes alcances incluindo, por vezes, subáreas no interior das disciplinas: estudos culturais, antropologia dos sistemas simbólicos, sociologia do conhecimento, sociologia da inovação, teoria de poder simbólico de Bourdieu, etnometodologia, interacionismo simbólico, teoria da ação comunicativa, teoria da conversação, perspectiva ecossistêmica, abordagem biossocial, materialismo histórico dialético, psicologia sócio-histórica; teoria histórico-crítica, psicologia social de Pichon-Riviére, epistemologia da complexidade, teorias de análise de redes sociais, análise institucional, análise sistêmica, referencial da bioética cotidiana, leitura organizacional da psicossociologia francesa contemporânea, teorias sociais da estigmatização, modelos mentais-mapas mentais e teoria da argumentação de Perelman.

A interdisciplinaridade que nutre a pesquisa qualitativa e a área das Ciências Sociais e Huma- 
nas em Saúde favorece a pluralidade e a heterogeneidade nas diversas orientações teóricas e metodológicas, assim como a proliferação de taxonomias e nomenclaturas. Em artigo recente, Bosi 10 utiliza o mito bíblico da Torre de Babel para comentar a dificuldade que essa realidade pode colocar para o diálogo entre os pesquisadores e apontar a impossibilidade de qualquer exercício de classificação taxionômica das teorias vigentes.

As "categorias metodológicas" foram consideradas aquelas utilizadas como ferramentas analíticas para interpretação do campo representacional e simbólico. Não foram aqui analisadas as categorias teóricas, empregadas para a definição e a operacionalização empírica do objeto de estudo.

Mais de uma categoria foi comumente listada no mesmo artigo, muitas elencadas como uma rede de sinonímia nem sempre condizente com suas definições teóricas de origem nas Ciências Sociais e Humanas em Saúde. Um artigo poderia, por exemplo, citar como categorias de seu estudo as "representações, percepções e crenças".

As categorias definidas como "percepção" (18\%), "significados/significação" (11\%) e "sentidos" (11\%), reunidas, foram predominantes, com 108 menções (40,6\% do acervo). A seguir, as categorias mais frequentes foram as "práticas", com 41 menções. Os "discursos e práticas discursivas" receberam nada mais que 24 citações. As representações, outrora tão frequentes nos estudos da área, foram vinculadas a apenas 22 artigos.

As "percepções” não necessariamente refletiam algum estudo de base fenomenológica, tampouco as categorias de "significados" distinguiam-se do emprego de "sentidos". Vale ressaltar que raramente as categorias declaradas eram definidas ou instituídas em um campo teórico no decorrer do texto. Exceção feita às categorias de "representação social" e as pouco citadas "habitus", "scripts sexuais”, "trocas linguísticas", "construção social do corpo" e "redes semânticas", que usualmente eram acompanhadas de teorização mais densa.

Categorias que apareceram em menos de dez artigos foram agrupadas no item "outras", constituindo imensa diversidade (117 enunciados). Aquelas mais ligadas à dimensão cognitiva de "entendimento", "conhecimento", “opinião" constaram desse grupo.

Por fim, os artigos sem quaisquer menções às categorias analítico-metodológicas constituíram, mais uma vez, a maioria no acervo (47 artigos; $17,6 \%$ ), revelando insuficiente descrição de métodos.

A utilização de mais de uma técnica para construção dos dados foi um traço marcante do acervo, de modo que $38,7 \%$ dos artigos recorreram a esta forma de triangulação. Considerando todas as citações, as entrevistas individuais foram as principais técnicas utilizadas, especialmente de tipo semiestruturado, seguidas pelas "observações", pesquisa documental e grupos focais. Apenas dois artigos não informaram o tipo de técnica empregada (Tabela 2).

Dos 70 artigos que declararam utilizar a "observação" como meio de produção empírica, 34 a definiram como "observação participante". Todavia, muito poucos teceram considerações acerca do nível de participação empreendida pelos autores, da construção do papel do investigador diante do grupo social envolvido, ou das limitações que sua filiação identitária impunha aos resultados do estudo.

Diante desses achados, é preciso problematizar a monotonia dos recursos empregados em face das inúmeras possibilidades de constituição de acervos para a análise sociocultural. Apenas 11\% dos artigos apontaram o uso de técnicas diferenciadas, tais como: grupos de discussão/reflexão; mapa de rede social; oficina de expressividade; lista de termos; modelo de decisão etnográfica; registro de conversas; coletânea de imagens (de embalagens de cigarros), gravação de reunião terapêutica, registro e revisão de prescrições de

\section{Tabela 2}

Distribuição dos artigos de Ciências Sociais e Humanas em Saúde segundo técnicas e procedimentos de análise, 2009-2010

\begin{tabular}{lcc}
\hline Técnicas e procedimentos & $\mathbf{n}$ & $\%$ \\
\hline Técnicas de produção de dados & & \\
$\quad$ Entrevistas individuais & 194 & 42,8 \\
Triangulação de técnicas & 103 & 22,7 \\
Observação & 70 & 15,5 \\
Pesquisa documental & 47 & 10,4 \\
Grupos focais & 37 & 8,2 \\
Sem informação & 2 & 0,4 \\
Procedimentos de análise & & \\
Análise de conteúdo & 113 & 42,5 \\
Análise de discurso & 16 & 6,0 \\
Hermenêutica dialética & 12 & 4,5 \\
Discurso do sujeito coletivo & 6 & 2,3 \\
Análise narrativa & 8 & 3,0 \\
Análise fenomenológica & 4 & 1,5 \\
Sem definição & 34 & 12,8 \\
Outras & 34 & 12,8 \\
Sem informação & 39 & 14,7 \\
Total & 266 & 100,0
\end{tabular}


medicamentos; oficinas; análise de postagens de weblogs; roda de conversa, filmagem; planografia (análise fotográfica).

Finalmente, o exame dos "procedimentos de análise" apontou uma indiscutível predominância da análise de conteúdo, sobretudo na vertente da análise temática. Os artigos sem quaisquer informações sobre como foi executada a interpretação dos dados também se destacaram, seguidos daqueles que descreveram o passo a passo dos procedimentos utilizados sem, no entanto, nomeá-los conforme algum método. Esta última opção é qualitativamente distinta, pois indica o domínio do processo interpretativo e uma possível recusa ou inadequação aos modelos existentes. As demais vertentes analíticas foram pouco enunciadas, mesmo aquelas de ampla tradição nas Ciências Sociais e Humanas em Saúde, tais como a "análise de discurso/análise de práticas discursivas", “análise fenomenológica”, análise de narrativas e as diferentes formas de hermenêutica. A categoria "outras" agrupou aquelas formas de análise citadas por menos de cinco artigos, como: análise semântica, análise de conversação, análise compreensiva, modelo comportamental, análise argumentativa, entre outros. Destaquese que métodos criados por autores brasileiros - "discurso do sujeito coletivo" (6) e uma nova modalidade de análise de conteúdo denominada "método da interpretação dos sentidos" (3) - começam a despontar.

Vários dos estudos analisados utilizam as técnicas de produção dos dados qualitativos de forma instrumental, caracterizando-se por um empirismo ateórico. Estes estudos, em geral, utilizam a análise de conteúdo temática e não desenvolvem conceitualmente as categorias que utilizam para abordar o universo simbólico. As falas dos sujeitos entrevistados são tomadas como verdades e descoladas das práticas e dos contextos socioculturais em que eles estão inseridos. Padecem, assim, do que Bourdieu et al. 5 denominam de ilusão da transparência.

O uso de softwares para o processamento dos dados ainda é muito pouco utilizado pelos autores, aparecendo em apenas 16 artigos. A ferramenta mais usada foi o NVivo (10 artigos), além do uso episódico do Evoc, Tri-deux-mots, Atlas e Ethnograph.

Com base nesses dados, seguimos com algumas reflexões e inferências, naturalmente abertas à arena de confrontação dialógica.

A análise apontou, em primeiro lugar, para uma considerável fragilidade de descrição da metodologia dos estudos apresentados. Podemos, inicialmente, pensar que se trata de "falta de espaço” editorial para maiores detalhamentos. De fato, as Ciências Sociais e Humanas em
Saúde são provenientes de tradições escritas, em que a discursividade e a construção argumentativa sofrem diante do espaço mais exíguo e sintético dos artigos. Consideramos, entretanto, que esta hipótese, embora plausível, não é suficiente, pois, mesmo no acervo por nós analisado, ainda que em minoria, houve estudos que apresentaram considerável densidade, indicando a exequibilidade de textos de alta qualidade no formato de artigo. A segunda hipótese, mais grave, apontaria para a deficiência de formação teórico-metodológica dos autores, praticantes desta ampla e heterogênea área denominada Ciências Sociais e Humanas em Saúde em saúde. Esse aspecto indica a necessidade de reflexão acerca da oferta e da qualidade desses conhecimentos nos cursos de graduação e pós-graduação em Saúde Coletiva, como já apontaram outros autores 2,10.

O acervo analisado nos sugere considerável monotonia e dominância de poucos tipos de modalidades de análise e de técnicas de produção de dados, reduzindo sobremaneira o amplo arsenal de escolhas e os novos e inovadores investimentos no percurso investigativo. A ideia que se constrói é a de que as Ciências Sociais e Humanas em Saúde não usufruem ainda da plenitude das ferramentas analítico-metodológicas de que dispõem, sejam as mais consagradas, sejam as mais recentes.

O conjunto dos artigos nos mostra ainda, em vários casos, o uso inadequado ou empiricista de certas categorias analíticas e mesmo de técnicas e métodos, fatos já observados por outros autores 10,11,12. Corrobora-se, assim, a hipótese das fragilidades de formação.

Apesar deste cenário que demanda cuidadosa reflexão, pudemos encontrar trabalhos inspiradores, apontando inovações na abordagem dos temas e objetos tratados. Destacam-se novos objetos de observação participante (centros de pesquisa, ciberespaço), inovações metodológicas que incluem a participação dos sujeitos no processo de construção e interpretação dos dados, a triangulação de métodos, incluindo o uso de imagens. Também merece menção a utilização de técnicas e procedimentos de análise dos dados, ainda pouco explorados no contexto atual das Ciências Sociais e Humanas em Saúde, a exemplo da análise de discurso, análise de conversação ou análises narrativas e hermenêuticas. 


\section{Resumo}

O presente artigo visa a mapear os usos teórico-metodológicos correntes nas pesquisas do subcampo das $\mathrm{Ci}$ ências Sociais e Humanas em Saúde, refletindo criticamente sobre eles. Foram selecionadas, por amostra de conveniência, três revistas brasileiras da área da saúde coletiva. Com base na leitura de 1.128 resumos publicados nos anos de 2009 e 2010, foram selecionados 266 artigos que apresentavam base empírica de investigação com aporte nos métodos oriundos das Ciências Sociais e Humanas em Saúde. O acervo composto foi classificado conforme as temáticas "referencial teóricometodológico", "tipo de estudo-desenho metodológico", "categorias analíticas utilizadas", "técnicas para a produção de dados" e "procedimentos de análise". Analisamos as categorias êmicas do acervo, valendo-nos das declarações literais dos autores. Todas as classificações e respectivas variáveis foram tabuladas em Excel. A maior parte dos artigos se autodenominou qualitativa e utilizou mais de uma técnica para a produção dos dados. Observou-se grande variedade de referenciais teóricos em contraste com a quase total predominância de um único tipo de análise de dados (análise de conteúdo). Em vários casos, identificaram-se lacunas importantes na exposição da metodologia da pesquisa e utilização instrumental das técnicas e métodos de pesquisa qualitativos. Destacam-se, porém, novos objetos de estudo e inovações teórico-metodológicas.

Ciências Sociais; Ciências Humanas; Pesquisa

\section{Referências}

1. Bourdieu P. O campo científico. In: Ortiz R, organizador. Pierre Bourdieu: Sociologia. São Paulo: Editora Ática; 1983. p. 122-55. (Coleção Grandes Cientistas Sociais).

2. Nunes ED, Ferreto LE, Oliveira ALO, Nascimento JL, Barros NF, Castellanos MEP. O campo da Saúde Coletiva na perspectiva das disciplinas. Ciênc Saúde Coletiva 2010; 15:1917-22.

3. Canesqui A. Sobre a presença das ciências sociais e humanas na saúde pública. Saúde Soc 2011; 20: 16-21.

4. Luz M. Especificidade da contribuição dos saberes e práticas das ciências sociais e humanas para a Saúde. Saúde Soc 2011; 20:22-31.

5. Bourdieu P, Chamboredon JC, Passeron JC. A profissão de sociólogo, preliminares epistemológicas. Petrópolis: Editora Vozes; 1999.

6. Camargo Jr. KR, Coeli CM, Caetano R, Maia VR. Produção intelectual em saúde coletiva: epistemologia e evidências de diferentes tradições. Rev Saúde Pública 2010; 44:394-8.

\section{Colaboradores}

Os dois autores coletaram e analisaram os dados, além de redigirem o artigo.

\section{Agradecimentos}

Agradecemos a Ana Maria Rico e Eliane Luz pela participação no processo de coleta e classificação de dados.
7. Minayo MCS. O desafio do conhecimento. 9a Ed. São Paulo: Editora Hucitec; 2006.

8. Gil AC. Como elaborar projetos de pesquisa. $5 \underline{a} \mathrm{Ed}$. São Paulo: Editora Atlas; 2008.

9. Denzin N, Lincoln Y. Introdução. A disciplina e a prática da pesquisa qualitativa. In: Denzin N, Lincoln Y, organizadores. O planejamento da pesquisa qualitativa. Porto Alegre: Editora Artmed; 2006. p. 15-41.

10. Bosi MLM. Pesquisa qualitativa em saúde coletiva: panorama e desafios. Ciênc Saúde Coletiva 2012 17:575-86.

11. Nakamura E. O método etnográfico em pesquisas na área da saúde: uma reflexão antropológica. Saúde Soc 2011; 20:95-103.

12. Victora C. Uma ciência replicante: a ausência de uma discussão sobre o método, a ética e o discurso. Saúde Soc 2011; 20:104-12.

Recebido em 14/Jul/2012 Aprovado em 23/Jul/2012 